Cahiers de recherches médiévales

\title{
Orléans entre 1350 et 1429
}

vue par quelques chroniqueurs de la fin du Moyen Âge

Françoise Michaud-Fréjaville

\section{CpenEdition}

Journals

Édition électronique

URL : https://journals.openedition.org/crm/718

DOI : $10.4000 / \mathrm{crm} .718$

ISSN : 1955-2424

Éditeur

Honoré Champion

\section{Édition imprimée}

Date de publication : 1 juin 2005

Pagination : 5-10

ISSN : $1272-9752$

Référence électronique

Françoise Michaud-Fréjaville, « Orléans entre 1350 et 1429 », Cahiers de recherches médiévales [En ligne], 12 spécial | 2005, mis en ligne le 28 juin 2008, consulté le 15 décembre 2022. URL : http:// journals.openedition.org/crm/718; DOI : https://doi.org/10.4000/crm.718 


\section{rir $\mathrm{RM}$}

\section{$-1-$ \\ Orléans entre 1350 et 1429, vue par quelques chroniqueurs de la fin du Moyen Âge}

Le héraut Berry, rappelant l'intervention des troupes royales arrivant avec Jeanne d'Arc, évoque «la bonne cité d'Orléans» et se réjouit de ce «que Dieu, à prière des saints, ne voulut pas que ladite cité fust prinse ne perye $»^{1}$. Ces saints étaient, bien entendu, les évêques saint Aignan et saint Euverte, dont on sait que la légende, entretenue peut-être par un clerc prenant ses distances avec le miracle johannique, voulait qu'ils soient apparus aux Anglais sur la muraille au cours du siège ${ }^{2}$. Ville épiscopale, bonne cité, Orléans est une des illustrations des différents caractères de la «bonne ville» des historiens d'aujourd'hui: une communauté d'habitants ${ }^{3}$, convoquée aux États, voire recevant ces États ${ }^{4}$, participant aux dépenses de guerre, même au prix de la liberté de certains de ses bourgeois ${ }^{5}$, enfin protégée par une enceinte fortifiée élevée et entretenue par des levées de tailles ou des droits sur le vin ou le sel' ${ }^{6}$. Orléans est la parfaite illustration de ce que Claude de Seyssel évoquait: «une bonne ville ou place forte bien ravitaillée, artillée et fournie de toutes choses nécessaires pour soutenir un siège et nourrir une garnison et un secours, est le sauvement de tout un royaume $»^{7}$.

C'est à partir de cette constatation que j'ai entrepris de rechercher ce que disaient les chroniqueurs au sujet de la ville d'Orléans à la fin du Moyen Âge, espérant

${ }^{1}$ Gilles le Bouvier dit le Héraut Berry, Chroniques du roi Charles VII, éd. H. Courtauld, L. Celier et M.-H. Jullien de Pommerol, Paris, 1973 (Société de l'histoire de France), p. 133.

${ }^{2}$ Boucher de Molandon, «La Délivrance d'Orléans et l'institution de la fête du 8 mai », Mémoires de la Société Archéologique et Historique de l'Orléanais, t. XVIII, p. 280 : «En icelui temps fut récité par aucuns des Anglois estans pour lors audit siège, avoir veu [...] deux prelas en abbit pontifical aller et circuir en cheminans sur les murs de la ville d'Orleans ». À vrai dire, cela était également une façon de comparer les Anglais aux pires barbares, les Huns de 451 !

${ }^{3}$ Bene populata de l'aveu des habitants en 1372 (cf. P. C. Timbal, La guerre de Cent ans vue au travers des registres du Parlement de Paris, Paris, 1960, p. 193).

${ }^{4} \mathrm{Ce}$ fut le cas en 1439: «Le roi avoit mandé toute ladite compaignie qui estoit pour ceste heure assemblée audit lieu d'Orléans pour ung chacun adviser au bien de la chose publicque » (cf. Gilles le Bouvier, op. cit., p. 207).

${ }^{5}$ Orléans fut contrainte d'envoyer deux otages en Angleterre (Jodoin à la Geule et Arnoul Regnier), en garantie de la rançon de Jean le Bon.

${ }^{6} \mathrm{La}$ ville obtint en mars 1385 la confirmation de son administration, élue pour lever les tailles afin de pouvoir fortifier la ville et réparer les portes démantelées après les émeutes de 1382 (cf., R. de Maulde, «De l'organisation municipale coutumière au Moyen Âge. Chartes municipales d'Orléans et de Montargis", Nouvelle revue de droit français et étranger, t. VIII, 1883, p. 1-40 et. J. Thibault, Orléans à la fin du Moyen Âge, vers 1380-vers 1450, thèse de doctorat dact., Paris IV, 1997, t. II, p. 479-494).

${ }^{7}$ Claude de Seyssel, La monarchie en France [...], cité par B. Chevalier, Les bonnes villes de France du XIV $V^{e}$ au XVI $I^{e}$ siècle, Paris, 1982, p. 54.

Cahiers de Recherches Médiévales (XII -XV S.), 12spé, 2005 
trouver ainsi non pas tant une image institutionnelle, mais l'écho d'une réputation, celle de la grande ville, de la forteresse, voire de l'université. Ce que l'on en disait pouvait-elle la distinguer des autres cités, être un reflet d'une quelconque originalité, d'une personnalité urbaine?

La quête fut décevante: il n'y a pratiquement jamais de description ou d'épithète accompagnant les mentions d'Orléans avant le siège, et c'est encore Froissart qui se montre le plus prolixe. Citant la ville en plusieurs occasions, il permet de dresser la liste des aspects que l'on retrouve à l'occasion chez d'autres acteurs, c'est pourquoi j'ai pratiquement laissé la parole à ce seul guide.

Orléans qui êtes au pays de Loire

La situation ligérienne est l'élément descriptif fondamental de la ville, la Loire est un obstacle naturel qui délimite dans le royaume des espaces humains. Quand en 1345 menace la chevauchée du duc de Derby, Philippe VI convoque l'ost à Orléans : «Si se assemblèrent cil signeurs et leurs gens en la cité d'Orliens et la environ, voires cil de par deça le Loire; et cil de dela[...]». La ville est un des points de passage obligé entre deux parties du territoire: lorsque les Français se lancent à la poursuite du Prince noir, l'été de 1456, «les gens du roi Jehan avoient passé la Loire ou pont d'Orliens", dit une des versions ${ }^{8}$. Une autre rapporte : «et passerent la riviere de Loire ensy qu'ils venoient, li aucuns a Orliens, li aultres a Mehun, li plusieurs a Saumur $"$. Le fleuve est la voie du voyage et du ravage, la ville le point de repère. Pendant les durs mois de 1358-1359 durant lesquels Robert Knowles et ses émules menèrent des troupes dans la région, «ils roboyent tout le pays entre le riviere de Loire et le riviere de Sainne, parquoy nuls n'osoit aller entre Paris et Vendomme, ne entre Paris et Orliens $»^{10}$. Et le chroniqueur ajoute : «ils rançonnoient toutes les villes [...] d'une part et d'autre de le riviere, de Nevers a Orliens $»^{11}$, ce qui semble plus juste que ce qu'en dit Jean le Bel ${ }^{12}$. Pour le Bascot de Mauléon, interrogé longtemps après ses aventures de jeune routier, c'est spontanément que se fait le rapprochement entre le fleuve et la ville: «Seguin de Batefol tenoit $[\ldots]$ dessoubs Loire jusqu'à Orliens $»^{13}$.

Orléans, ville forte

Si l'on reste étonné que les chroniqueurs passent sous silence le rôle universitaire de la ville, l'activité parfois brouillonne de ses écoliers ou étudiants, pourtant

\footnotetext{
${ }^{8}$ Jean Froissart, Chroniques, éd Kervyn de Lettenhove, t. V, p. 382.

${ }^{9} I b i d$., t. XVII (version abrégée), p. 343. De fait, au XIV ${ }^{\mathrm{e}}$ siècle, entre Orléans et Saumur, outre Meung-sur-Loire ici citée, il existait également un pont à Beaugency (B. Toulier, «Le pont de Beaugency (Loiret)», Bulletin de la Société Archéologique et Historique de l'Orléanais, 1979, n 52, p. 43), ainsi qu'à Blois, Amboise et Tours.

${ }^{10}$ Ibid., t. VI, p. 34.

${ }^{11}$ Ibid. , p. 112.

${ }^{12}$ Jean le Bel, Chronique, éd. J. Viard et E. Desprez, Paris 1905 (Société de l'histoire de France), t. II p. 286 : «Robert Canoll [...] mist tous ces pays a subjection et a sa volenté sans nul debat, de Orlians jusqu'à Notre-Dame du Puys ».

${ }^{13} I b i d .$, t. XI, p. 12.
} 
fustigée par l'opinion publique ${ }^{14}$, brocardée par les poètes et les méchantes langues ${ }^{15}$ et protégée par la justice ${ }^{16}$, on ne peut ignorer que la cité élève des murailles audessus du plat pays. La chevauchée de Robert Knowles et de ses émules, nous l'avons vu, était une occasion de rappeler que la fonction de refuge était fondamentale. Les campagnes s'étaient vidées : «les gens du plat pays s'estoyent tout retraicts à Paris ou a Orliens, car tres petites d'autres villes en celle province se tenoient contre ces routiers $"{ }^{17}$. Si les murailles sont bonnes, elles n'enserrent, hélas, pas toutes les maisons et «tous les fourbours d'Orlyens furent ars et essilles par force gens, jusques as portes $»^{18}$.

\section{L'espace orléanais}

De fait, les mentions, toujours brèves, ne donnent de la ville aucune véritable description, ne proposent aucune individualisation. Et, en effet, avant le siège de 1429, la cité n'existait guère par elle-même pour nos chroniqueurs, mais plutôt comme partie d'un ensemble de points géographiques qui dessinent l'aire d'action des troupes amies ou ennemies. Elle appartient aux itinéraires, elle scande un territoire particulier, elle est un des éléments d'une énumération, d'ailleurs signifiante.

En 1356, «le roi fit un mandement que [ils] venirent devers lui, à Chartres, à Orliens et à Blois $»^{19}$, et en se lançant à la suite des Anglais, les troupes royales passèrent le fleuve à "Orlians, a Meun, a Saumur, a Blois et a Tours, et la ou ils pooient $»^{20}$. Les parcours des exactions du Gallois Ruffin sont définis par les distances entre Paris et Vendôme, Paris et Orléans, Paris et Montargis, Paris et Chartres. Les bonnes villes ici distinguées sont les «bien gardées », les autres courent le risque d'être pillées. C'est en des termes semblables que sont évoquées les bandes de routiers de 1369 qui «desrobent le pays [...] mesmement entre Paris et Orliens, entre Paris et Chartres, et en le conté de Blois $»^{21}$. Enfin, et pour ne pas allonger trop les citations, quand, dans une des versions de Froissart, en 1380, le duc d'Anjou accourt

\footnotetext{
${ }^{14}$ Les rixes furent nombreuses entre bourgeois et les écoliers. Les Orléanais prétendaient que tout ce qu'ils retiraient des plaintes qu'ils formulaient était l'obligation de faire amende honorable.

${ }^{15} \mathrm{C}$. Vulliez, «Le temps des études universitaires à travers l'exemple orléanais des derniers siècles du Moyen Âge », dans Les entrées dans la vie, initiations et apprentissages, XII Congrès des historiens médiévistes de l'Enseignement supérieur public (Nancy, 1981), Annales de l'Est, 1982, $\mathrm{n}^{\circ} 1-2$, p. 150. L'auteur cite les vers célèbres du Miroir de mariage d'Eustache Deschamps : "Po estudient, bien se battent / pour leurs fillettes se combattent", filles ou vin, ou les deux, n'aidant pas au calme universitaire...

${ }^{16}$ Le procès-verbal de l'enquête des commissaires du roi sur le retour des écoles à Orléans en 1320 après leur exil volontaire, est dithyrambique, il vante : «le grand fruit et plantureux profit qui genereument, et non pas tant seulement en notre royaume mais par le monde, souloit dériver et issir de l'estude d'Orliens de qui, senz cesse, deffluoient li ruisseaux de science qui universseaument s'estendoient» (M. Fournier, Les statuts et privilèges des Universités françaises, Paris, 1890, t. I, Orléans, p. 49).

${ }^{17}$ Jean Froissart, op. cit., t. VI, p. 35.

${ }^{18}$ Ibid., t. VI, p. 112-113. (1356)

${ }^{19}$ Ibid., t. V, p. 382.

${ }^{20}$ Ibid.

${ }^{21}$ Ibid., t. V, p. 347.
} 
au chevet de Charles V mourant, son itinéraire passe également par «Tours en Tourraine, par Bloys, et par Orliens a Paris [...] Car il entendoit que li rois, ses freres, agrevoit moult $\aleph^{22}$.

De façon nette se dessine ainsi à travers ces mentions, un espace limité au sud par la Loire, à l'est par la route d'Orléans à Paris et à l'ouest par une ligne Tours-Chartres-Paris. C'est le triangle de Beauce, le pays constamment associé à Orléans, même lorsque aucune nécessité ne l'impose, itinéraire, voyage ou tournoiement de troupes. Dès qu'apparaît le nom d'Orléans, un rapprochement suggère son intégration au vieil espace capétien. Robert Briquet, premier compagnon du Bascot, meurt «en Orlénois entre le païs de Blois et la terre au duc d'Orlians, en une place qu'on dit Olivet $~^{23}$. Y a-t-il un projet de débarquement en Angleterre (1386) ? «S'avaloirent les gens de Touraine, d'Orliens, de Gastonois, de Beausse »" ${ }^{24}$ Part-on en Castille? «Si se départirent les chevaliers de Thouraine, d'Orliens, de Blois, de Beausse $»^{25}$. Pour nous, habitués aux cartes routières, bien plantés au cœur de nos points cardinaux, ces relations spatiales sont évidentes mais à quelles images correspondaient-t-elles pour les lecteurs du XIV ${ }^{\mathrm{e}}$ siècle ? La ville, la bonne ville que nous voyons comme un point à la jonction de la RN 20, de l'autoroute et de la Loire, comment écrivains et lecteurs pouvaient-ils se la figurer?

\section{La ville du siège}

Jusqu'à l'encerclement par les troupes de Salisbury, les lecteurs qui n'avaient pas eux-mêmes une fois dans leur vie franchi le pont ne «voyaient» pas la ville et n'avaient sans doute aucune idée sûre de sa configuration, mais l'aventure qui tint en haleine, semble-t-il, une bonne partie du royaume et peut-être de l'Europe, a provoqué une série de descriptions destinées à faire comprendre aux lecteurs, ou à rappeler aux acteurs, la situation militaire et les actions d'éclat d'un épisode majeur de la guerre.

Thomas Basin retrouve pour qualifier la ville, «remplie de toutes sortes de richesses et peuplée plus que beaucoup d'autres d'un grand nombre d'habitants $»^{26}$, les formules des bourgeois orléanais de $1372^{27}$, mais sa science va bien au-delà. Il rappelle que la cité ne s'étend que sur une seule des rives, ce qui est exact si on considère que seule est «ville» la forteresse emparée, et que ce ne fut que dans un second temps que la tête du pont sur la rive opposée, avec son entrée fortifiée, fut occupée. Basin souligne également les faubourgs détruits avec leurs églises. Il relève, avant Jacques Le Goff, l'importance de la ville par la présence des quatre couvents des

\footnotetext{
${ }^{22}$ Ibid., t. IX, p. 288.

${ }^{23}$ Jean Froissart, Chroniques, éd. P. Ainsworth et A. Vavaro, livres III et IV, Paris, Librairie générale française (Lettres gothiques), 2004, livre III, p. 210. Olivet, prieuré de Marmoutier, (com. et cant., arr. Orléans, Loiret) sur la rivière du Loiret ne doit pas être confondu avec l'abbaye cistercienne d'Olivet (act., com. de Saint-Julien-sur-Cher, can. Mennetou-sur-Cher, arr. Romorantin-Lanthenay, Loir-et-Cher), alors au diocèse de Bourges.

${ }^{24}$ Jean Froissart, Chroniques, éd. Kervyn de Lettenhove, t. XII, p. 9.

${ }^{25}$ Ibid., p. 67.

${ }^{26}$ Thomas Basin, Histoire de Charles VII, éd C. Samaran, Paris, 1964, t. I, p. 116 : statuerant ipsam Aurelianensem urbem aggredi, opum abundencia et popularum ante multas alias tunc frequencia ac numerositate refertam.

${ }^{27}$ P. C. Timbal, op. cit., p. 293.
} 
ordres mendiants ${ }^{28}$. Cependant cette description est bien postérieure aux événements et n'est, en fin de compte, guère plus évocatrice ou mieux placée dans l'espace que la courte notation de Clément de Fauquenbergue quand celui-ci intègre dans son journal la déroute anglaise du 7 mai avec l'Anglais Glasdale dans « la tour de l'yssue du pont par dela de la Loyre »- par rapport à Paris où se trouve notre greffier - et «les autres capitaines [...] tenans le siege et les bastilles par deça Loyre, devant la ville d'Orliens $»^{29}$.

Si Jean Lefèvre indique, lui aussi, l'existence de la «tour du boult du pont», il situe le siège du "couté devers le Solonge » et néglige de signaler le complet encerclement de la ville ${ }^{30}$. Gilles le Bouvier, le héraut Berry, insiste sur l'isolement de la cité auquel sont parvenus les ennemis en l'inscrivant dans une large ceinture de villes prises à l'automne 1428, une sorte de deuxième ligne de bastilles renforçant celle qui enserre étroitement les assiégés : «En l'an mil CCCC vingt et huit, fut mis le siège à Orléans et y mis des bastilles des costés de Beausse et de Solongne, et prit Yenville en Beausse [...], Meun-sur-Loire, Boisgency, Jargueau, La Ferté de Gaules, Puviers en Gastinois [...] ; et tint Sully de par le conte de Salisbury un chevalier de Nivernois $»^{31}$. Sur la topographie ou les caractéristiques de la ville, en revanche, le héraut est d'une précision fort médiocre, considérant sans doute que les lecteurs savent où se trouvent « la bastille au bout du pont» où fut frappé à mort Salisbury et la «bastille de Saint Leu», celle des Augustins et une imaginaire bastille de la Vicomté 32 .

Jean Chartier, comme les autres, structure l'espace orléanais entre le «costé devers la Beausse", si détruit que "gens a cheval pouvoient aller franchement partout où estoient assises [...] les églises et faulxbourgs $»^{33}$, et le coté devers Soloigne ", où il met en relief le pont et son boulevard, lieux de l'exploit de Jeanne et théâtre de la délivrance.

La situation de charnière entre deux «pays » paraît finalement la caractéristique la plus éclatante de la ville. De son pont, tout à la fois articulation et verrou entre le nord et le sud du royaume, elle a tiré des avantages - commerce et population - et subi les inconvénients - le siège et les destructions. C'est sans doute tout ce que sauront d'Orléans les copistes et lecteurs des chroniques du $\mathrm{XV}^{\mathrm{e}}$ siècle.

${ }^{28}$ Cum suburbanam haberet perampla, utpote in quibus essent quatuor conventus ordinum mendicancium (Thomas Basin, op. cit., ibid.). Les quatre couvents étaient les Cordeliers (franciscains) et les Jacobins (dominicains) au nord, les Carmes à l'ouest et les Ermites de Saint-Augustin sur la rive sud, à la tête du pont.

${ }^{29}$ Journal de Clément de Fauquenbergue, éd. A. Tuetey, Paris, 1909 (Société de l'histoire de France), t. II, p. 307.

${ }^{30}$ Chronique de Jean le Fèvre, seigneur de Saint-Remy, éd. F. Morand, Paris, 1881 (Société de l'histoire de France), t. II, p. 140.

${ }^{31}$ Gilles le Bouvier, Chronique..., p. 130-131. La Ferté de Gaules, act. La Ferté-Saint-Aubin com. et cant., arr. Orléans, ici «de Gaules» du nom de Jean de Gaules, maréchal du duc d'Orléans, mort à Azincourt (cf. L. Monnier, Notice sur Jean de Gaules, Arch. dép. Loiret, dact., 1961), était également appelée La Ferté-Nabert (Chronique de la Pucelle, éd. Vallet de Viriville, Paris, 1848 , t. I, p. 60). Puviers $=$ Pithiviers.

${ }^{32}$ Gilles le Bouvier, op. cit., p. 131.

${ }^{33}$ Jean Chartier, Chronique de Charles VII, éd. Vallet de Viriville, Paris, 1848, t. I, p. 60. 
Sortie un bref instant de la litanie des villes d'entre Seine et Loire, Orléans n'en reçoit pas pour cela de qualificatifs particuliers, elle n'est ni héroïque, ni martyre, ni encore miraculée ou autres épithètes dont nous sommes si peu avares aujourd'hui. Modeste entre les bonnes villes, elle rentre dans l'ordre, en ayant cependant chanté à elle-même sa gloire dans le Journal du siège ${ }^{34}$ et dans le Mystère $d u$ siège $e^{35}$, et mis en place puis pérennisé une fête commémorative, sa liturgie d'action de grâce, sa prière privée à laquelle ne sont pas alors conviés des spectateurs mais seulement des participants. Pour les chroniqueurs et les mémorialistes, il n'y a plus rien à dire, la ville disparaît de leurs textes quand ils continuent plus avant dans les récits de l'histoire de leur temps.

${ }^{34}$ Journal du siège d'Orléans 1428-1429, augmenté des comptes de ville 1429-1431, éd. P. Charpentier et C. Cuissard, Orléans, 1896.

${ }^{35}$ Le Mistere du siège d'Orléans, éd. critique V. L. Hamblin, Genève, Droz, 2002 (Textes littéraires français). 